\title{
Pattern and Risk Factors for Retinal Vein Occlusion in Onitsha, Nigeria
}

\author{
Sebastian N N Nwosu
}

Guinness Eye Center, Onitsha, Nigeria

\section{SUMMARY}

Objectives: To determine the pattern and risk factors for retinal vein occlusion at the Guinness Eye Center, Onitsha, Nigeria.

Materials and Methods: Case files of all patients with retinal vein occlusion between May 1997 and April 2004 were reviewed. Information on age, sex, visual acuity, ocular complications and associated risk factors were abstracted into a standard proforma and analysed.

Results: 45 patients (48 eyes) were seen with a mean age of 58 years; range - 41-77 years. There were 16 (35.6\%) male patients and $29(64.4 \%)$ female patients. Thirty-eight $(84.4 \%)$ patients $(40$ eyes) had central retinal vein occlusion (CRVO), 7 (15.6\%) patients (8 eyes) had branch vein occlusion (BRVO), and $29(60.4 \%)$ eyes (all CRVO) had ischaemic vein occlusion. Twenty-eight (58.3\%) eyes were blind, while 18 (37.5\%) had visual impairment. Twenty-five $(55.6 \%)$ patients had hypertension, 10 $(22.2 \%)$ were diabetic and $10(22.2 \%)$ had glaucoma.

Conclusions: Retinal vein occlusion is an important retinal vascular disease that causes visual loss. The incidence of the disease could be reduced if the associated risk factors were detected and treated early. A prospective study was carried out to clearly identify the modifiable risk factors for the disease in our environment.

Key words: retinal vein occlusion, incidence, pattern, risk factors, Nigeria

\section{INTRODUCTION}

Retinal vein occlusion is a retinal vascular disorder with potentially blinding complications. Worldwide, central retinal vein occlusion along with branch retinal vein occlusion constitute the second commonest retinal vascular disease, after diabetic retinopathy. ${ }^{1,2}$ Reports on eye diseases from different parts of Nigeria have documented the contribution of retinal vein occlusion to ocular morbidity. ${ }^{3-5}$ Abiose $^{3}$ and Ayanru, in earlier studies, separately reported the rarity of retinal vascular diseases, including retinal vein occlusion in Nigeria. A hospital-based study in Anambra State, however, showed that retinal vein occlusion constitutes $12.5 \%$ of retinal diseases. ${ }^{5}$

During the period under review, the management of retinal vein occlusion at the Guinness Eye Center consisted of prescribing low doses of aspirin in the hope of decreasing blood platelet aggregation; and treatment of any known systemic and or ocular disease known to predispose to the disease. Laboratory investigations were limited to packed cell volume and platelet counts, as the centre's laboratory at that time lacked the capacity to estimate serum lipids and cholesterol. Facilities for fluorescein angiography and laser treatment were not available.

This study was aimed at determining the pattern and possible risk factors for retinal vein occlusion at a tertiary eye centre, as data on this important disease in Nigeria is scarce.

\section{MATERIALS AND METHODS}

This is a retrospective study. The case files of all consecutive new patients with retinal vein occlusion seen at the Guinness Eye Center, Onitsha over a 7-year-period (May 1997 - April 2004) were reviewed.

Information on age; gender; habits; visual acuity; clinical diagnosis, including type of vein occlusion, ocular complications, and ocular and systemic risk factors were abstracted into a standard proforma and analysed. Blindness was defined as visual acuity $<3 / 60$, while acuity $<6 / 18-3 / 60$ was regarded as visual impairment.

\section{RESULTS}

During the 7-year period, 43 new patients (48 eyes) with retinal vein occlusion were seen. Five patients had bilateral disease. The age range of the patients was 41-77 years; with a median of 66 years and a mean of 58 years (table 1$)$. There were $17(39.5 \%)$ male and $26(60.5 \%)$ female patients.

${ }^{*}$ Correspondence: Sebastian N N Nwosu, Guinness Eye Center, PMB 1534, Onitsha Nigeria. Email: sabenwosu@yahoo.com 
Twenty patients $(46.5 \%)$ sniffed tobacco powder ('snuff') while $8(18.6 \%)$ drank alcohol. The time interval from the onset of symptoms to presentation at the hospital ranged from 1 to 18 months with a mean of 5 months.

\begin{tabular}{lcccc}
\hline Table 1. Age and sex distribution & & & \\
\hline Age (Years) & M & F & Total & $\%$ \\
\hline $41-50$ & 2 & 6 & 8 & 18.6 \\
$51-60$ & 5 & 3 & 8 & 18.6 \\
$61-70$ & 8 & 10 & 18 & 41.9 \\
$\geq 71$ & 2 & 7 & 9 & 20.9 \\
Total & 17 & 26 & 43 & 100.0 \\
\hline
\end{tabular}

Thirty-six patients $(83.7 \%)$ had central retinal vein occlusion (CRVO), while branch retinal vein occlusion (BRVO) occurred in 7 patients (16.3\%). Bilateral disease occurred in 4 patients with CRVO and in a patient with BRVO. Thus, a total of 48 eyes of 43 patients had retinal vein occlusion. In 29 eyes $(60.4 \%)$, the disease was ischemic. All the ischemic cases occurred in CRVO patients.

The presenting visual acuity ranged from 6/12 to NPL (table 2). Eighteen eyes (37.5\%) had visual impairment and 28 eyes $(58.3 \%)$ were blind. Twenty-two of the $28(78.6 \%)$ blind eyes had ischemic vein occlusion. The 2 blind eyes with acuity of no light perception (NPL) had neovascular glaucoma secondary to rubeosis iridis and angle neovascularization.

Table 2. Presenting acuity (affected eye)

\begin{tabular}{lcc}
\hline Acuity & No & $\%$ \\
\hline $6 / 12$ & 1 & 2.1 \\
$6 / 18$ & 1 & 2.1 \\
$6 / 24$ & 6 & 12.5 \\
$6 / 36$ & 2 & 4.1 \\
$6 / 60$ & 7 & 14.6 \\
$3 / 60$ & 3 & 6.3 \\
$<3 / 60-\mathrm{LP}$ & 26 & 54.2 \\
NPL & 2 & 4.1 \\
Total & 48 & 100.0 \\
\hline
\end{tabular}

Chronic simple glaucoma was co-existent in 10 patients (23.2\%); diabetes mellitus in 10 patients (23.2\%); and hypertension in 25 patients $(58.1 \%)$. While 29 patients $(67.4 \%)$ did not keep more than one follow up appointment, $13(30.2 \%)$ failed to keep any follow up appointment at all.

\section{DISCUSSION}

The results of this study show that retinal vein occlusion is an important cause of low vision among the afflicted in our hospital. There were more female patients among the study cohort. Hospital-based studies are affected by selection bias. Records show that more adult females than adult males usually consult at the Guinness Eye Center, Onitsha. All the patients in this cohort were adults. The female preponderance in this cohort may be a reflection of the pattern of attendance at the hospital.

On the other hand, oral contraceptives are known to predispose users to vein occlusion. ${ }^{6}$ Women are more likely to use oral contraceptives and hormone replacement therapy. However, the use of these drugs was not documented in any of our patients. A prospective study is required to better define the role of such drugs in the aetiology of vein occlusion in Nigerians.

Glaucoma, found in $23.2 \%$ of our patients, could predispose to vein occlusion. ${ }^{1}$ Conversely, ischemic central retinal vein occlusion is a cause of neovascular glaucoma. ${ }^{1}$ Hypertension and diabetes mellitus were the two systemic risk factors for the disease recorded in our patients. A study in a major teaching hospital in the United States of America reported that patients with retinal vein occlusion also had a higher incidence of hypertension and diabetes mellitus. ${ }^{7}$ The course of these ocular and systemic risk factors could be modified by treatment. Therefore, vigorous treatment of the ocular and systemic risk factors is recommended as a way of perhaps preventing the occurrence of the disease. Nearly half of our patients take snuff, a mixture of tobacco and potash powder. The possible role of this powdery mixture in the aetiology of retinal vein occlusion is at present not clear and requires further study.

This study was limited by the lack of facilities for fluorescein angiography in our hospital. The advantages of fluorescein angiography in the treatment of these patients include clearly defining the areas of ischemia, determining the rate of blood flow through retinal vessels, better detection of macula oedema, and improved monitoring of treatment outcome. ${ }^{8,9,10}$ The Central Retinal Vein Occlusion Study recommends laser treatment when there is at least 10 disc areas of retinal nonperfusion. In our hospital, the diagnosis of ischemic vein occlusion is made on clinical grounds using such parameters as relative afferent pupillary defect. It is therefore difficult to objectively determine the number of disc areas of capillary nonperfusion.

More recent advances include the use of optical coherent tomography in the diagnosis and in monitoring the treatment outcome of macular oedema. ${ }^{11}$ McAllister and Constable recommended laser-induced chorioretinal anastomosis for the treatment of perfused central retinal vein occlusion. ${ }^{12}$ The intravitreal injection of triamcinolone acetonide has recently been found to be useful in the treatment of macula oedema, especially the refractory type $^{13}$ Neither laser photocoagulation nor intravitreal 
steroids was used on our patients, as they were not available during the period under review.

Another limitation of the study is the high default rate among the patients. This prevented us from determining the final visual outcome, the full impact on the burden of blindness, and the natural history of retinal vein occlusion among Nigerians. Loss of a high percentage of patients to follow up has been a source of concern to many workers in Nigeria, and the Guinness Eye Center is not exempt. In the case of retinal vein occlusion patients, the lack of facilities for proven effective treatment may have contributed to the problem of default. Nevertheless, retinal vein occlusion as a cause of low vision and blindness is well demonstrated in this study in which none of the affected eyes had visual acuity better than $6 / 12$ and more than $58 \%$ of the eyes were blind when first seen at the hospital.

In conclusion, the incidence of retinal vein occlusion in our hospital is relatively low; and central retinal vein occlusion is more common. Women are more likely to be affected than men. Based on presenting visual acuity, the disorder impacts negatively on vision, increasing low vision and blindness, albeit mainly uniocular. Patients, therefore, need to be educated on the nature of the disease, including the known risk factors and the natural history. The need to provide facilities for the investigation and effective treatment of the disease cannot be overemphasized.

Finally, a prospective study is required to further define the epidemiological and clinical characteristics of patients with retinal vein occlusion in our environment.

\section{REFERENCES}

1. Ciardella AP, Clarkson JG, Guyer DR, Renno RZ, Yannuzzi LA. Central Retinal Vein Occlusion: A primer and review. 286307.

2. Finkelstein D. Branch retinal vein occlusion. In Guyer DR et al., eds. Retina-Vitreous-Macula. Philadelphia: WB Saunders Company 1999: 308-315.
3. Abiose A. Retinal diseases in Nigerians - A preliminary report. Niger Med J 1978; 6(2): 180-189.

4. Ayanru JO. Environment, culture and eye diseases. (Experience in Benin-City, Bendel State of Nigeria). Proc. African Eye Seminar. Nairobi, Kenya: Merck, Sharpe \& Dohme Int. 1982: 41-63.

5. Nwosu SNN. Prevalence and pattern of retinal diseases in Guinness Eye Hospital, Onitsha, Nigeria. Ophthalmic Epidemiol 2000; 7: 41-48.

6. Stowe GC III, Zakov ZN, Albert DM. Central retinal vein occlusion associated with oral contraceptives. Am J Ophthalmol 1978; 86: 789.

7. Elam MG, Bhatt AK, Quinlan PM, Enger C. The risk for systemic vascular diseases and mortality in patients with central retinal vein occlusion. Ophthalmology 1990; 97: 15431548 .

8. Mandova N, Guyer DR, Yannuzzi LA, Nichol J, Orlock D. Principles of fluorescein angiography. In Guyer DR et al., eds. Retina-Vitreous-Macula. Philadelphia: WB Saunders Company 1999: 29-38.

9. The Central Vein Occlusion Study Group. A randomized clinical trial of early panretinal photocoagulation for ischemic central retinal vein occlusion. Ophthalmology 1995; 102: 14341444.

10. The Branch Retinal Vein Occlusion Study Group. Argon laser scatter photocoagulation for prevention of neovascularization and vitreous hemorrhage in branch retinal vein occlusion. Arch Ophthalmol 1986; 104: 34-41.

11. Hee MR, Puliafito CA, Wong C, et al. Quantitative assessment of macular edema with optical coherence tomography. Arch Ophthalmol 1995; 113: 1019-1029.

12. McAllister I, Constable I. Laser-induced chorioretinal anastomosis for treatment of non-ischemic central retinal vein occlusion. Arch Ophthalmol 1995; 113: 456-462.

13. Greenberg PB, Martidis A, Rogers AH et al. Intravitreal triamcinolone acetonide for macular edema due to central retinal vein occlusion. Br J Ophthalmol 2002; 86: 247-248. 